23

\title{
Индуцируемые напряжения, возникающие в кристаллическом кремнии при воздействии ультракоротких лазерных импульсов различной длительности в воздухе и воде
}

\author{
(C) Н.А. Смирнов, С.И. Кудряшов, Н.Н. Мельник, П.М. Папилова, И.А. Шерстнев, А.А. Ионин, Ц. Чэнь
}

Физический институт им. П.Н. Лебедева РАН,

119991 Москва, Россия

e-mail: cna1992@mail.ru

Поступила в редакцию 08.06.2021 г.

В окончательной редакции 23.06.2021 г.

Принята к публикации 25.06.2021 г.

\begin{abstract}
Производилась модификация поверхности кремния в одноимпульсном режиме фемто-пикосекундными лазерными импульсами (0.3 и $10 \mathrm{ps})$ ближнего ИК диапазона $(1030 \mathrm{~nm})$ при абляции в воздухе и воде. Полученные структуры изучались с помощью микроскопии комбинационного рассеяния света. В ходе исследования установлено, что на границе кратера появляются нанокристаллиты размером 7-8 nm. Обнаружены локальные механические напряжения в центре кратера, знак которых зависит от приложенной плотности энергии. Наибольшие локальные сжимающие напряжения возникают в воде в субфиламентационном режиме при максимальных плотностях энергии.
\end{abstract}

Ключевые слова: кремний, спектроскопия комбинационного рассеяния света, ультракороткие импульсы, одноимпульсная абляция в воздухе и в жидкости, локальные напряжения.

DOI: $10.21883 /$ OS.2021.10.51501.2399-21

\section{Введение}

Лазерная абляция является одним из основных методов в прецизионной обработке во многих технологических операциях, в частности в микроэлектронике, где кремний на сегодняшний день является базовым элементом. Очень важно, чтобы для обработанных лазером, а также близлежащих областей не менялся химический и фазовый состав, а также отсутствовали напряжения в материале. Для данных целей наиболее походящими являются лазерные ультракороткие импульсы (УКИ, длительность менее $10^{-10} \mathrm{~s}$ ), которые имеют наименьшую область термического воздействия на материал и все чаще применяются для лазерной прецизионной микрообработки [1-4].

Локальные напряжения, возникающие после лазерной обработки, критически влияют на структурную надежность полупроводниковых устройств [5-7]. Поэтому важно охарактеризовать остаточное напряжение и оптимально выбрать параметры лазерного излучения, при которых возникающие напряжения в кремнии минимальны.

Спектроскопия комбинационного рассеяния света (КРС) позволяет идентифицировать материал и дает информацию о фононной частоте, электрон-фононном взаимодействии, примеси, кристаллической структуре, ориентации кристаллов и механической деформации в материале [5-10]. Локальность данного метода при фокусировке объективами с большой числовой апертурой составляет менее $1 \mu \mathrm{m}$, что позволяет нам исследовать полученные лазерным путем структуры в поперечном сечении. Кремний хорошо изучен с точки зрения спектроскопии КРС. Хорошо известно, что пик фундаментального колебания кристаллического кремния расположен на частоте $521 \mathrm{~cm}^{-1}$. При аморфизации кремния спектр КРС представляет собой широкую бесструктурную полосу с максимумом $\sim 480 \mathrm{~cm}^{-1}$ [6]. Любые изменения и деформации, вызванные давлением в материале, будут приводить к сдвигу частоты собственного колебания в твердом теле и определяться для кремния по формуле $[8,9]$

$$
\sigma(\mathrm{M \Pi а})=-435\left(\omega-\infty_{0}\right)\left(\mathrm{cm}^{-1}\right),
$$

где $\omega$ - положение пика кремния в ненапряженном состоянии, $\omega_{0}$ - положение пика кремния в напряженном состоянии. Если происходит разупорядочение решетки, то полуширины полос КРС увеличиваются $[11,12]$. При уменьшении размеров кристаллов до размеров $\sim 2-10 \mathrm{~nm}$ происходит нарушение правил отбора по волновому вектору, и максимум полосы КРС смещается в сторону низких частот, а сама полоса становится асимметричной: появляется низкочастотное крыло основного колебания $[13,14]$. Из такого спектра по форме и положению линии КРС в области $520 \mathrm{~cm}^{-1}$, отвечающей за колебания $\mathrm{Si}-\mathrm{Si}$, можно оценить размер нанокристаллов.

Известно большое количество работ, в которых производился анализ индуцируемых лазером структур на поверхности кремния, в том числе и при одноимпульсном лазерном воздействии $[15,16]$. При этом в данных работах нет детального анализа полученных структур в 


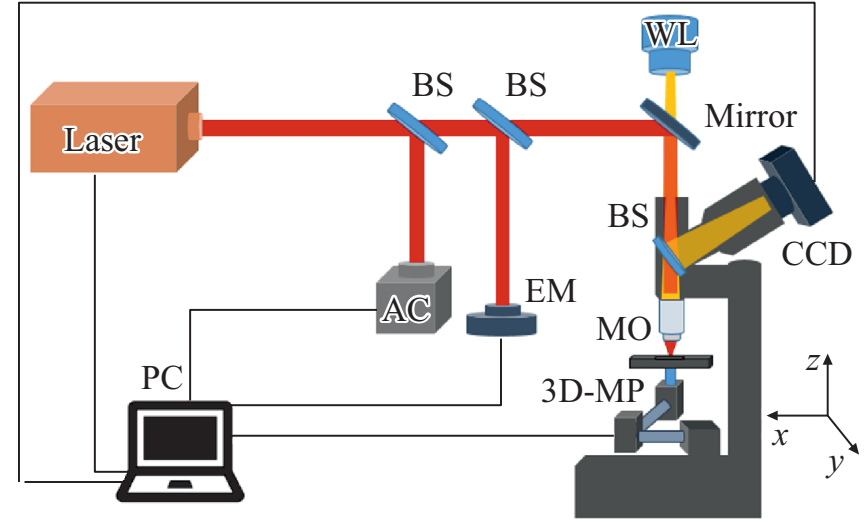

Рис. 1. Схема экспериментальной установки: Laser-лазер Satsuma, SHG - генератор второй гармоники, BS - делитель луча, ЕМ - измеритель энергии, АС - автокоррелятор, $\mathrm{MO}-$ микрообъектив с числовой апертурой $N_{A}=0.25, \mathrm{WL}-$ источник белого света, 3D-MP - трехосевая моторизованная платформа.

рамках спектроскопии КРС в зависимости от плотности энергии в импульсе.

В настоящей работе исследовались кратеры, полученные путем облучения кремниевой пластины лазерными ультракороткими импульсами варьируемой длительности $(0.3$ и $10 \mathrm{ps})$ в воздушной и водной средах. Характеризация кратеров производилась с помощью метода микроспектроскопии КРС.

\section{Экспериментальная часть}

В эксперименте производилась абляция кремниевой пластины (толщина $380 \mathrm{~nm}$, ориентация поверхности 111) в одноимпульсном режиме. В качестве буферной среды во время абляции использовались воздух и дистиллированная вода. Слой воды над поверхностью образца составлял 2-3 $\mathrm{mm}$.

Схема эксперимента показана на рис. 1, $a$. В качестве источника лазерного излучения в работе был использован волоконный лазер Satsuma (Amplitude Systemes) c активной средой на ионах $\mathrm{Yb}^{+3}$ (длина волны основной гармоники $1030 \mathrm{~nm}$, частота следования импульсов 0-2 MHz). Исследование кратеров производилось для длительностей 0.3 и 10 ps. Энергия УКИ в ТЕМ00моде плавно менялась с помощью тонкопленочного отражательного ослабителя. Лазерное излучение фокусировалось на поверхность образца через объектив с числовой апертурой $N_{A}=0.25$ с фокусным расстоянием $7.50 \mathrm{~mm}$ в пятно $3.2 \mu \mathrm{m}$ по уровню интенсивности гауссова пучка $1 / \mathrm{e}$.

Спектры КРС регистрировались на спектрометре U1000 производства фирмы ИСА Жобен-Ивон (Франция). Прибор снабжен оптическим микроскопом, позволяющим оптическим способом выделять исследуемую поверхность образца размером до $1 \times 1 \mu \mathrm{m}$. В качестве
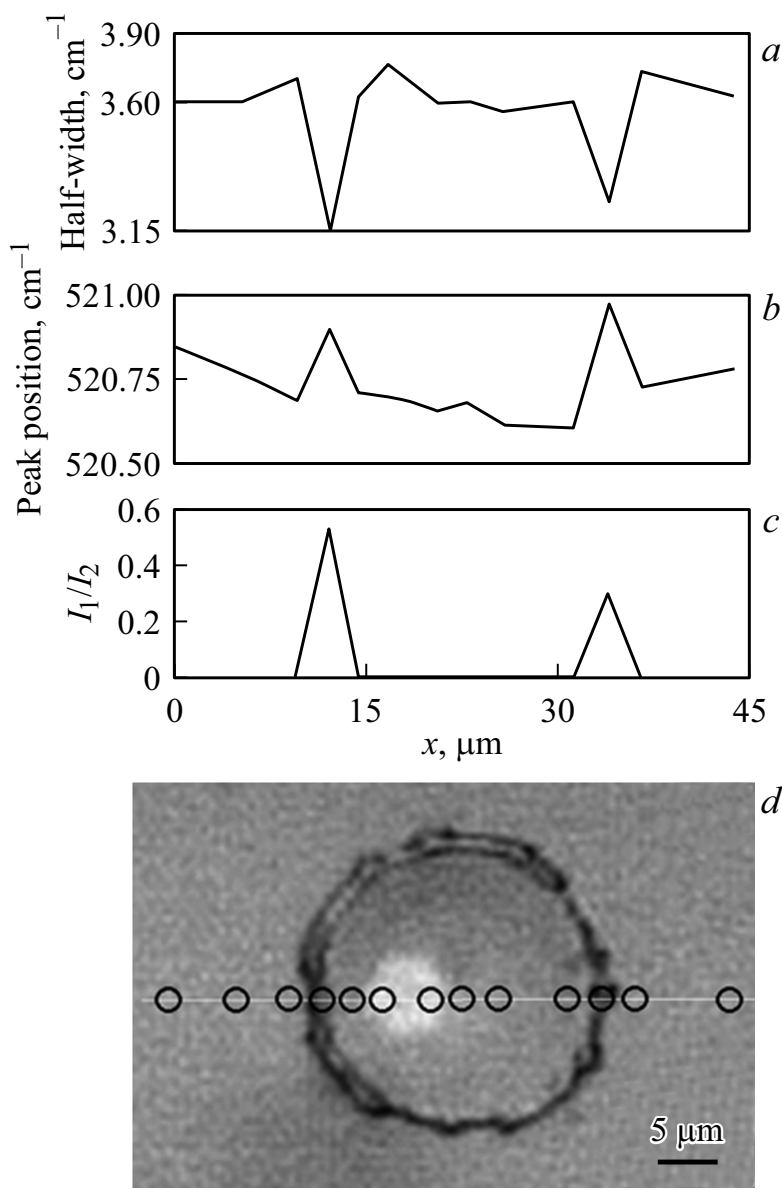

Рис. 2. Изменение свойств пика КРС фундаментального колебания $\mathrm{Si}$ от места регистрации: $a-$ зависимость полуширины; $b-$ зависимость частоты; $c-$ проявление асимметрии пика, $d-$ фотография образца, на которой отмечены точки регистрации спектров КРС.

источника лазерного излучения использовался аргоновый лазер $(514.53 \mathrm{~nm})$. Мощность выходного излучения составляла не менее $50 \mathrm{~mW}$. Все измерения образцов выполнялись в геометрии „на отражение“. Для объектива с числовой апертурой $N_{A}=0.95$ и используемой длины волны размер фокусировки (диаметр пятна $D$ ) будет равен [5]

$$
D=\frac{0.61 \lambda_{0}}{N_{A}}
$$

Глубину проникновения лазерного излучения в кремний $D_{p}$ можно рассчитать по формуле [17]

$$
D_{p}=\frac{2.3}{2 \alpha\left(\lambda_{0}\right)},
$$

где $\lambda_{0}$ - длина волны возбуждающего излучения, $\alpha$ - коэффициент поглощения для данной длины волны $\left(14.96 \mathrm{~cm}^{-1}\right)$. Полученные значения: $D \approx 0.63 \mu \mathrm{m}$, $D_{p} \approx 0.77 \mu \mathrm{M}$. 

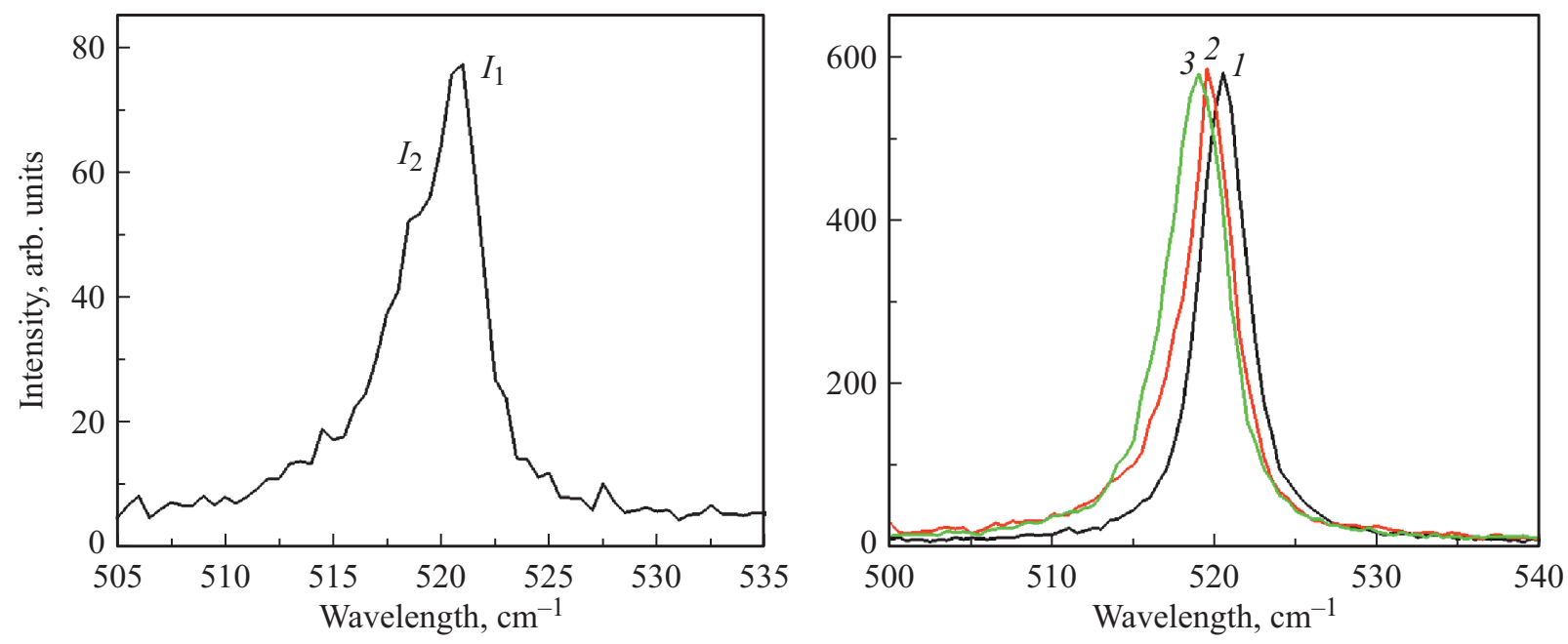

Рис. 3. $a-$ спектр КРС на границе кратера; $b$ - спектры КРС от разных точек границы кратера (кривые 2,3) и исходного монокристалла (1).
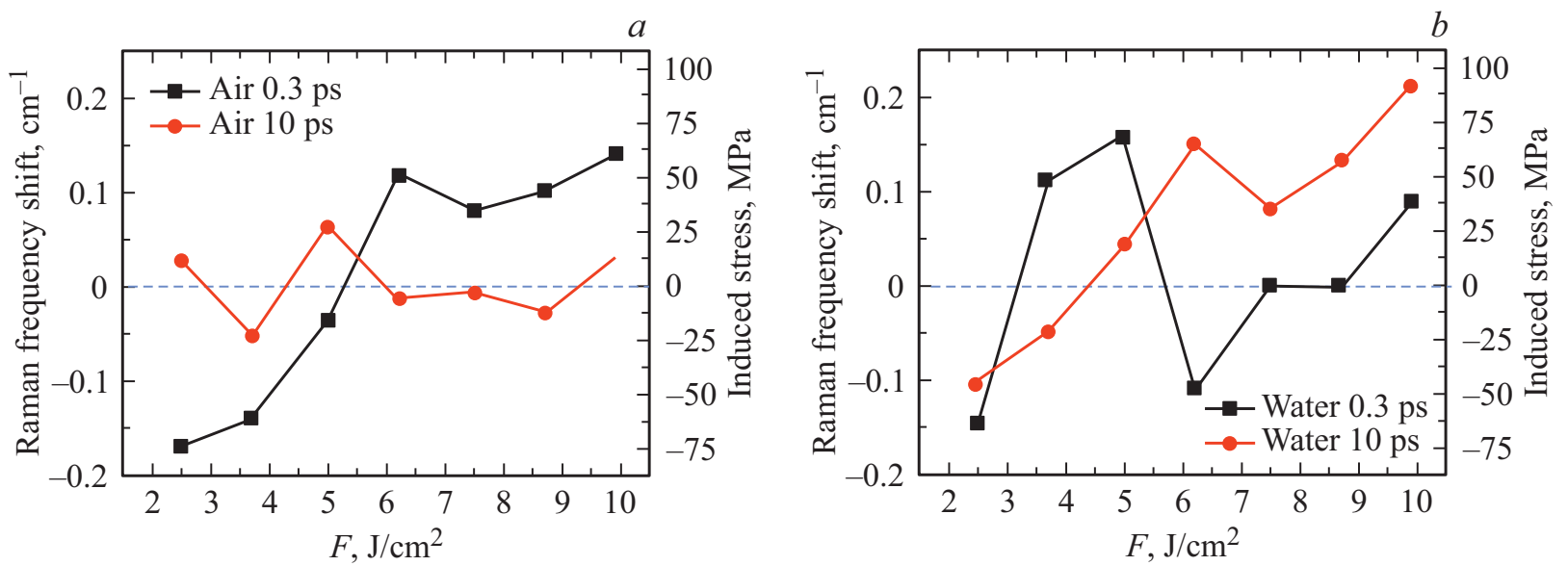

Рис. 4. Сдвиги основного пика относительно чистого кремния от энергии для длительностей импульса 0.3 и 10 ps при абляции: $a$ - на воздухе, $b-$ в воде.

\section{Результаты и обсуждения}

Для кратеров на кремнии были исследованы спектры КРС в области $400-560 \mathrm{~cm}^{-1}$. Спектры были получены для нескольких областей кратеров. Это бортик (край), область внутри и вне кратера. При исследовании кратера с тонкой переходной границей возникла проблема. При последовательном сканировании образца, для того чтобы с уверенностью попасть пятном лазерного возбуждения на границу, шаг сканирования должен быть меньше, чем толщина границы и диаметр пятна лазера (которые приблизительно равны). Для этого потребуется слишком много времени. Для решения данной проблемы нами использована следующая методика. Вручную пятно лазерного возбуждения совмещалось с интересующей областью образца. После регистрации спектра КРС изображение образца в поле микроскопа регистрировалось и запоминалось. Процесс повторялся для всех измерений. Далее, созданная нами программа определяла сдвиг одного изображения относительно другого, и, таким образом, мы получали карту образца и точки на ней, где регистрировались спектры КРС. На рис. 2, $d$ представлено оптическое изображение кратера, на котором отмечены точки, где регистрировались спектры КРС.

Из графиков для полуширины (рис. 2,a) и частоты (рис. $2, b)$ видно, что полуширина пика практически не меняется, а положение максимума уменьшается по частоте в центре кратера. Отметим, что форма пиков всегда, кроме границы, симметрична. Это объясняется возникновением локального напряжения при лазерной обработке.

Непосредственно на границе кратера (рис. 2, $a, b$ ) мы видим уменьшение полуширины и увеличение частоты пика КРС кремния. Происходит это из-за того, что для пика КРС в данной области появляется асимметрия в форме, и мы производим аппроксимацию по двум плечам данного пика (рис. 3,a). Для качественной де- 
монстрации асимметрии мы аппроксимировали полосы КРС двумя симметричными полосами и на графике отложили отношение интенсивностей этих полос (рис. 2,c). Это чисто качественный подход. Поэтому и данные для графиков рис. $2, a, b$ в точках, соответствующих границам кратера, не имеют особого смысла.

Рассмотрим подробно спектры КРС непосредственно на границе кратера. Как уже было сказано, для данной области мы всегда наблюдаем асимметрию формы полосы КРС фундаментального колебания кремния (рис. 3,a). Из данного спектра также видно, что полоса является составной - здесь явно присутствует полоca $521 \mathrm{~cm}^{-1}$ монокристаллического кремния и асимметричная полоса, имеющая меньшую частоту.

Это происходит потому, что ширина границы кратера и диаметр пятна возбуждающего излучения близки, и одновременно могут регистрироваться как граница кратера, так и монокристаллические области. Для более корректной регистрации спектра КРС от границы были выбраны кратеры, полученные при наибольшей плотности энергии и имеющие наибольшую толщину бортика. На рис. $3, b$ приведены примеры таких спектров. Сдвиг и появившуюся асимметрию главного пика мы связываем с возникновением нанокристалитов в этой области. Рассчитать размер нанокристаллитов можно по сдвигу $\Delta \omega$ фононной моды кремния [18]:

$$
\Delta \omega(D)=-A\left(\frac{\alpha}{D}\right)^{\gamma}
$$

где $\Delta \omega(D)$ - сдвиг полосы КРС в наноструктурах диаметром $D, \alpha$ - постоянная решетки $\mathrm{Si}(0.543 \mathrm{~nm})$, $D$ - диаметр наноструктуры, $\gamma=1.44$ - параметры аппроксимации, описывающей локализацию фонона в нанометрических сферах диаметром $D, A-$ эмпирическое значение $\left(47.74 \mathrm{~cm}^{-1}\right)$. Использование этой формулы и полученные данные по сдвигу полос КРС дает оценку $D \sim 7-8 \mathrm{~nm}$.

Было произведено сравнение сдвигов полос КРС кремния в центре кратеров в зависимости от длительности и энергии (рис. 4). Расчет возникающих напряжений производился по формуле (1). Минимальное воздействие проявилось при минимальной интенсивности лазерного импульса (10 ps на воздухе), при котором от энергии напряжения меняются слабо и находятся в пределах $13^{-22} \mathrm{MPa}$. При высоких плотностях энергии (более $5 \mathrm{~J} / \mathrm{cm}^{2}$ ) наблюдается сдвиг пика КРС в длинноволновую область для абляции на воздухе для $0.3 \mathrm{ps}$ и в жидкости $10 \mathrm{ps,} \mathrm{что} \mathrm{соответствует} \mathrm{возникновению}$ сжимающих напряжений в приповерхностном слое. Значения напряжения при максимальной плотности энергии доходит до $91 \mathrm{MPa}$. При этом для данных условий, но при меньших плотностях энергии пик сдвигается в коротковолновую область, которой соответствует растягивающие напряжения, при пересчете составляющие $-63 \mathrm{MPa}$.

Для абляции в жидкости (рис. 4, $b$ ) при длительности импульса 0.3 ps и при плотностях энергии выше $5 \mathrm{~J} / \mathrm{cm}^{2}$ мы в работе [19] наблюдали возникновение филаментации при критической мощности лазерного излучения ₹ 4-6 MW, которая уменьшает эффективную плотность энергии, доходящей до поверхности мишени. Отображение данного эффекта мы наблюдаем для возникающих напряжений в кремнии. При максимальной энергии $\left(9.9 \mathrm{~J} / \mathrm{cm}^{2}\right)$ возникают сжимающие напряжения, которые в два раза меньше, чем при абляции на воздухе. Скачок локального напряжение в области $5 \mathrm{~J} / \mathrm{cm}^{2}$ можно связать переходом в субфиламентационный режим, дальнейшее уменьшение плотности энергии ведёт к уменьшению возникающих напряжений, а затем они становятся отрицательными. То есть зависимость в субфиламентационном режиме ведет себя так же, как и зависимости при абляции на воздухе для 0.3 ps и в жидкости для $10 \mathrm{ps}$.

При абляции в жидкости для 10 ps абляция производится в субфиламентационном режиме, и наблюдаются наибольшие сжимающие напряжения за счет того, что слой воды сдерживает расширение области с продуктами абляции, тем самым увеличивая амплитуду ударной волны [20].

\section{Заключение}

В настоящей работе производилась модификация поверхности кремния в одноимпульсном режиме фемтопикосекундными лазерными импульсами (0.3 и $10 \mathrm{ps})$ ближнего ИК диапазона $(1030 \mathrm{~nm})$ при абляции на воздухе и в воде. Полученные структуры изучались с помощью микроскопии КРС. Обнаружено, что на границе кратера появляются нанокристаллиты размером 7-8 nm. Обнаружены локальные механические напряжения, возникающие в центре кратера, знак которых зависит от приложенной плотности энергии. Наибольшие локальные сжимающие напряжения возникают в воде при субфиламентационном режиме при максимальных плотностях энергии. Минимальные напряжения возникают при абляции в воздухе при воздействии лазерным излучением с длительностью импульса 10 ps.

\section{Конфликт интересов}

Авторы заявляют, что у них нет конфликта интересов.

\section{Список литературы}

[1] Chichkov B.N. et al. // Appl. Phys. A. 1996. V. 63. N 2. P. 109-115. doi 10.1007/BF01567637

[2] Neuenschwander B. et al. // Phys. Proc. 2014. V. 56. P. 1047-1058. doi 10.1016/j.phpro.2014.08.017

[3] Smirnov N.A. et al. // JETP Letters. 2018. V. 108. N 6. P. 368-373. doi 10.1134/S002136401818011X

[4] Smirnov N.A. et al. // Opt. and Quant. Electron. 2020. V. 52. N 2. P. $1-8$. doi 10.1007/s11082-019-2169-1

[5] Sheremet E. et al. // Phys. Stat. Sol. (a). 2019. V. 216. N 19. P. 1900106. 
[6] Amer M.S., El-Ashry M.A., Dosser. L.R., Hix K.E., Maguire J.F., Irwin Bryan. // Appl. Surface Sci. 2005. V. 242. N 1-2. P. 162-167. doi 10.1016/j.apsusc.2004.08.029

[7] Ma L., Qiu W., Fan X. // Microelectronics Reliability. 2021. V. 118. P. 114045. doi 10.1016/j.microrel.2021.114045

[8] Wolf I.D. // J. Raman Spectrosc. 1999. V. 30. N 10. P. 877-883. doi 10.1002/(SICI) 1097-4555(199910)30:10< 877::AID-JRS464>3.0.CO;2-5

[9] Kang Y. et al. // Optics and Lasers in Engineering. 2005. V. 43. N 8. P. 847-855. doi 10.1016/j.optlaseng.2004.09.005

[10] Xing Ma L. et al. // AIP Advances. 2019. V. 9. N 1. P. 015010.

[11] Campbell I.H., Fauchet P.M. //Solid State Commun. 1986. V. 58. N 10. P. 739-741. doi 10.1016/0038-1098(86)90513-2

[12] Richter H., Wang Z.P., Ley L. // Solid State Commun. 1981. V. 39. N 5. P. 625-629. doi 10.1016/0038-1098(81)90337-9

[13] Periasamy S. et al. // Zeitschrift für Physikalische Chemie. 2017. V. 231. N 9. P. 1585-1598. doi 10.1515/zpch-20160961

[14] Viera G., Huet S., Boufendi L. // J. Appl. Phys. 2001. V. 90. N 8. P. 4175-4183. doi 10.1063/1.1398601

[15] Bonse J., Brzezinka K.W., Meixner A.J. // Appl. Surface Sci. 2004. V. 221. N 1-4. P. 215-230. doi 10.1016/S01694332(03)00881-X

[16] Kiani A., Venkatakrishnan K., Tan B. // Optics Express. 2009. V. 17. N 19. P. $16518-16526$. doi 10.1364/OE.17.016518

[17] De Wolf I. // Semiconductor Science and Technology. 1996. V. 11. N 2. P. 139.

[18] Periasamy S. et al. // Zeitschrift für Physikalische Chemie. 2017. V. 231. N 9. P. 1585-1598. doi 10.1515/zpch-2016-0961

[19] Smirnov N.A. etal. // Appl. Surface Sci. 2021. V. 562. P. 150243. doi 10.1016/j.apsusc.2021.150243

[20] Anisimov S.I. et al. // J. Physics: Conference Series. IOP Publishing. 2020. V. 1556. N 1. P. 012004. doi 10.1088/1742$6596 / 1556 / 1 / 012004$ 